\title{
Borsuk-Ulam Theorem and Maximal Antipodal Sets of Compact Symmetric Spaces
}

\author{
Bang-Yen Chen*
}

(Communicated by Kazım İlarslan)

\begin{abstract}
The Borsuk-Ulam theorem is a well-known theorem in algebraic topology which states that if $\phi: S^{n} \rightarrow \mathbb{R}^{k}$ is a continuous map from the unit $n$-sphere into the Euclidean $k$-space with $k \leq n$, then there is a pair of antipodal points on $S^{n}$ that are mapped by $\phi$ to the same point in $\mathbb{R}^{k}$.

In this paper we study continuous real-valued functions of compact symmetric spaces. The main result states that if $f: M \rightarrow \mathbb{R}$ is a continuous isotropic function of a compact symmetric space $M$ into the real line $\mathbb{R}$. Then $f$ carries some maximal antipodal set of $M$ to the same point in $\mathbb{R}$, whenever $M$ is one of the following spaces: Spheres; the projective spaces $\mathbb{F} P^{n}(\mathbb{F}=\mathbb{R}, \mathbb{C}, \mathbb{H})$; the Cayley plane $F I I$; the exceptional spaces $E I V ; E I V^{*} ; G I$; and the exceptional Lie group $G_{2}$. Some additional related results are also given.
\end{abstract}

Keywords: Borsuk-Ulam theorem; maximal antipodal set; continuous function; 2-number; compact symmetric space.

AMS Subject Classification (2010): Primary: 55M20; Secondary: 53C35.

\section{Introduction}

Many interesting theorems have been proved in topology about continuous maps defined on an $n$-sphere (see for instance [21]). The theorem with which we concern in this paper is the following well-known result in algebraic topology.

Borsuk-Ulam Theorem (BUT). Let $\phi: S^{n} \rightarrow \mathbb{R}^{k}$ be a continuous map of an $n$-sphere into the Euclidean $k$-space. If $k \leq n$, then $\phi$ maps some pair of antipodal points into a single point.

Clearly, the Borsuk-Ulam Theorem fails for a continuous map $\phi: S^{n} \rightarrow \mathbb{R}^{k}$ with $k>n$, because $S^{n}$ embedded in $\mathbb{R}^{n+1}$.

The Borsuk-Ulam Theorem has numerous applications which range from combinatorics to differential equations and even economics (cf. [18]).

For $n=1$, BUT implies that there always exist a pair of opposite points on the earth's equator with the same temperature.

For $n=2$, BUT implies that at any moment there is always a pair of antipodal points on the Earth's surface with equal temperatures and equal barometric pressures.

The Borsuk-Ulam Theorem was conjectured by S. Ulam at the Scottish Café in Lvov, Ukraine. The conjecture was solved in 1933 by K. Borsuk [2]. It turned out that the result had been proved three years before in [17] by L. Lusternik and L. Schnirelmann (see [2, footnote, page 190]). Since then, many alternative proofs as well of extensions have been found by various authors, as collected by H. Steinlein in [26].

In this paper, we seek Borsuk-Ulam's type results for compact symmetric spaces. The main result of this paper is related with maximal antipodal sets and the 2-numbers of compact symmetric spaces which were introduced about 35 years ago by B.-Y. Chen and T. Nagano in [11, 12].

Both notions of maximal antipodal sets and the 2-numbers have been studied by a number of mathematicians with some nice applications to several areas of mathematics. 


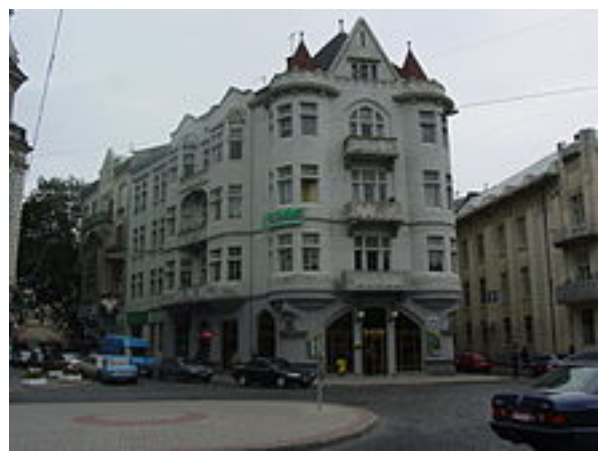

Figure 1. Scottish Café in Lvov, Ukraine

For the most recent surveys on 2-numbers and maximal antipodal sets of compact Lie groups and of compact symmetric spaces, please refer to $[7,8]$.

\section{Symmetric spaces}

The class of Riemannian manifolds with parallel Riemannian curvature tensor, that is, $\nabla R=0$, was first introduced independently by P. A. Shirokov in 1925 and by H. Levy in 1926. This class is known today as the class of locally symmetric Riemannian spaces.

It was É. Cartan who noticed in 1926 that irreducible spaces of this type are separated into ten large classes each of which depends on one or two arbitrary integers, and in addition there exist twelve special classes corresponding to the exceptional simple groups. Based on this, É. Cartan created his theory of symmetric Riemannian spaces in his famous papers

"Sur une classe remarquable d'espaces de Riemann"

in 1926 [4].

Symmetric spaces are generalizations of spaces of constant sectional curvature. Though symmetric spaces need not have constant sectional curvature, their sectional curvatures remain constant under parallel translations of 2-dimensional tangent planes along regular curves.

Symmetric spaces arise in a wide variety of situations in both mathematics and physics. They are important objects of study in representation theory and harmonic analysis as well as in differential geometry. Symmetric spaces are the most beautiful and most important Riemannian manifolds.

On the one hand, the class of symmetric spaces contains many prominent examples which are of great importance for various branches of mathematics, like compact Lie groups, Grassmannians and bounded symmetric domains. Any symmetric space has its own special geometry; euclidean, elliptic and hyperbolic geometry are only the very first examples. On the other hand, these spaces have much in common, and there exists a rich theory.

An isometry $s$ of a Riemannian manifold $M$ is said to be involutive if $s^{2}=i d_{M}$. A Riemannian manifold $M$ is a symmetric space if for each point $x \in M$ there is an involutive isometry $s_{x}$ of $M$ such that $x$ is an isolated fixed point of $s_{x}$. The involutive isometry $s_{x}$ is called the symmetry at $x$. From the point of view of Lie theory, a symmetric space is the quotient $G / K$ of Lie group $\mathrm{G}$ by a Lie subgroup $K$, where the Lie algebra $\mathfrak{k}$ of $K$ is also required to be the (+1)-eigenspace of an involution of the Lie algebra $\mathfrak{g}$ of $G$.

Denote by $G$ the closure of the group of isometries generated by $\left\{s_{p}: p \in M\right\}$ in the compact-open topology. Then $G$ is a Lie group which acts transitively on the symmetric space; hence the typical isotropy subgroup $K$, say at a point $o \in M$, is compact and $M=G / K$. Therefore, symmetric spaces are homogeneous spaces as well. From the point of view of the Erlangen program, in a homogeneous space one may understand that "all points are the same".

A Hermitian symmetric space is a Hermitian manifold which at every point has a symmetry preserving the Hermitian structure.

In this paper, we use standard symbols (as in [14]) to denote symmetric spaces, mostly. Here are a few minor exceptions. More specifically than $A I, A I(n)$ denotes $S U(n) / S O(n), A I I(n):=S U(2 n) / S p(n)$, etc. $G_{d}\left(\mathbb{R}^{n}\right), G_{d}\left(\mathbb{C}^{n}\right)$ and $G_{d}\left(\mathbb{H}^{n}\right)$ are the Grassmann manifolds of $d$-dimensional subspaces in the real, complex and quaternion vector spaces (or modules), respectively. $M^{*}$ denotes the bottom space (the adjoint space in [14]) 
of the space $M$. The standard notations for the exceptional spaces such as $G_{2}, F_{4}, E_{6}, \ldots, G I, \ldots, E I X$ denote the simply-connected spaces, where we write $G I$ for $G_{2} / S O(4)$.

Every complete totally geodesic submanifold of a symmetric space is a symmetric space. For a symmetric space $M$, the dimension of a maximal flat totally geodesic submanifold of $M$ is a well-defined natural number which is called the rank of $M$, denoted by $r k(M)$. It follows from the equation of Gauss that $r k(B) \leq r k(M)$ for each totally geodesic submanifold $B$ of a symmetric space $M$ (cf. [5]). Clearly, the rank of a symmetric space is at least one. It is well-known that the class of rank one compact symmetric spaces consists of $n$-sphere $S^{n}$, a projective space $\mathbb{F} P^{n}(\mathbb{F}=\mathbb{R}, \mathbb{C}, \mathbb{H})$, or the 16-dimensional Cayley plane $F I I=\mathcal{O} P^{2}$.

Suppose a finite group $\Gamma$ is acting on two spaces $M$ and $N$ freely as automorphism groups. Then $\Gamma$ acts on the product space $M \times N$ freely. And the orbit space $(M \times N) / \Gamma$ is called the dot product of $M$ and $N$ (with respect to $\Gamma$ ) and denoted by $M \cdot N$. In most cases $\Gamma$ will be the group of order two acting on $M$ and $N$ as the covering transformation groups for double covering morphisms in the sequel. $\Gamma$ will not be mentioned in that case, if $\Gamma$ is obvious or if $\Gamma$ need not be specified.

\section{3. $\left(M_{+}, M_{-}\right)$-theory}

Here we provide a brief introduction of the $\left(M_{+}, M_{-}\right)$-theory for compact symmetric spaces introduced by B.-Y. Chen and T. Nagano in [10,12]. Our approach to compact symmetric spaces was based on antipodal points and fixed point sets on compact symmetric spaces. Consequently, our approach to compact symmetric spaces is very different from other approaches done by Cartan and others.

In fact, our approach to compact symmetric spaces plays the Key Roles in the determination of 2-numbers of compact symmetric spaces; including the determination of 2-ranks of compact Lie groups (see [6, 7]).

Let $o$ be a point of a compact symmetric space $M=G / K$. We call a connected component of the fixed point set $F\left(s_{o}, M\right) \backslash\{o\}$ of the symmetry $s_{o}$ in $M$ a polar of $o$. We denote it by $M_{+}$or $M_{+}(p)$ if $M_{+}$contains a point $p$. We have the following useful lemma from [10] (see, also [6, page 15]).

Lemma 3.1. Let $M=G / K$ be a compact symmetric space. Then for each antipodal point $p$ of $o \in M$, the isotropy subgroup $K$ at o acts transitively on the polar $M_{+}(p)$. Moreover, we have $K(p)=M_{+}(p)$ and $K(p)$ is connected. Consequently, we have $M_{+}(p)=K / K^{p}$, where $K^{p}$ is given by $\{k \in K: k(p)=p\}$.

When a polar consists of a single point, we call it a pole.

Lemma 3.2. Under the hypothesis of Lemma 3.1, the normal space to $M_{+}(p)$ at $p$ in $M$ is the tangent space of a connected complete totally geodesic submanifold $M_{-}(p)$. Thus we have

$$
\operatorname{dim} M_{+}(p)+\operatorname{dim} M_{-}(p)=\operatorname{dim} M .
$$

Lemma 3.3. For each antipodal point $p$ of o in a compact symmetric space $M$, we have (1) $r k\left(M_{-}(p)\right)=r k(M)$ and (2) $M_{-}(p)$ is a connected component of the fixed point set $F\left(s_{p} \circ s_{o}, M\right)$ of $s_{p} \circ s_{o}$ through $p$.

Polars and meridians are totally geodesic submanifolds of a compact symmetric space $M$; they are thus compact symmetric spaces as well. Polars and meridians have been determined for every compact connected irreducible Riemannian symmetric space (see $[6,12,19,20])$. One of the most important properties of these totally geodesic submanifolds is that $M$ is determined by any pair of $\left(M_{+}(p), M_{-}(p)\right)$ completely.

Let $M$ be a compact connected Riemannian symmetric space and $o$ be a point in $M$. If there exists a pole $p$ of $o \in M$, then we call the set consisting of the midpoints of the geodesic segments from $o$ to $p$ the centrosome and denote it by $C(o, p)$. Each connected component of the centrosome of $\{o, p\}$ is a totally geodesic submanifold of $M$. Centrosomes play some important roles in topology. In particular, centrosomes were applied by J. M. Burns in [3] to compute homotopy in many compact symmetric spaces.

\section{Maximal antipodal sets and 2-numbers}

Two points $\{p, q\}$ on a Riemannian manifold $M$ are called antipodal if there exists a closed geodesic $\gamma$ in $M$ such that $p$ and $q$ are antipodal points in $\gamma$. A subset $S$ of $M$ is called an antipodal set if any two points in $S$ are antipodal. An antipodal set $A_{2} M$ of $M$ is a maximal antipodal set if it is not a proper subset of any antipodal set of $M$. A closed geodesic in a Riemannian manifold is simply called a circle. 
The notions of maximal antipodal sets and 2-numbers of connected Riemannian manifolds were introduced by B.-Y. Chen and T. Nagano in [11]. It was proved in [12] that the 2-numbers relates directly with the 2-rank of compact connected Lie group studied initially by A. Borel and J. P. Serre in [1]. A detailed study on 2-numbers and maximal antipodal sets of compact symmetric spaces, including compact Lie groups, was established in [12] (see also [6]).

For a compact symmetric space $M$, the 2-number denoted by $\#_{2} M$, is defined to be the maximal possible cardinality \# $A_{2} M$ of a subset $A_{2} M$ of $M$ such that the point symmetry $s_{x}$ fixes every point of $A_{2} M$ for every $x \in A_{2} M$. It is known that a subset $A_{2}$ of a connected symmetric space $M$ is antipodal if and only if any pair of points in $A_{2}$ are antipodal points on some closed geodesic in $M$.

The definition is equivalent to saying that $\#_{2} M$ is a maximal possible cardinality $\# A_{2} M$ of a subset $A_{2} M$ of $M$ such that, for every pair of points $x$ and $y$ of $A_{2} M$, there is a closed geodesic of $M$ on which $x$ and $y$ are antipodal to each other. Thus the invariant $\#_{2} M$ can also be defined on Riemannian manifolds. Because every compact Riemannian manifold admits a closed geodesic [16], the 2-number $\#_{2} M$ of a compact Riemannian manifold is at least 2 .

Obviously, the (Riemannian) $n$-sphere $S^{n}$ satisfies

$$
\#_{2} S^{n}=2 .
$$

Clearly, if $B$ is a complete totally geodesic submanifold of a compact symmetric space $M$, then every antipodal set of $B$ is also an antipodal set of $M$. Consequently, we have $\#_{2} B \leq \#_{2} M$. Moreover, for the (Riemannian) product of two compact symmetric spaces, we have

$$
\#_{2}(M \times N)=\left(\#_{2} M\right)\left(\#_{2} N\right) .
$$

Chen and Nagano proved in [12] many fundamental properties of maximal antipodal sets (see also, [6, 7]). They also explicitly described antipodal sets in most compact Riemannian symmetric spaces, but they did not mention antipodal sets in oriented real Grassmann manifolds. The antipodal sets in oriented real Grassmann manifolds was then studied and determined later by H. Tasaki in [32].

As it was pointed out in [12], the invariant, $\#_{2} M$, has certain bearings on the topology of $M$; for instance, $\#_{2} M$ equals $\mathcal{X}(M)$, the Euler number of $M$, if $M$ is a semisimple hermitian symmetric space. And in general one has the inequality $\#_{2} M \geq \mathcal{X}(M)$ for any compact connected symmetric space $M$.

Furthermore, M. Takeuchi proved in [27] that $\#_{2} M=\operatorname{dim} H\left(M, \mathbb{Z}_{2}\right)$ for any symmetric $R$-space, where $H\left(M, \mathbb{Z}_{2}\right)$ is the homology group of $M$ with coefficients in $\mathbb{Z}_{2}$.

It was proved in [30] that any two maximal antipodal sets of a symmetric $R$-space or of a Hermitian symmetric spaces are congruent,

The following theorem from [12] provides some link between dot product, centrosome and 2-numbers of compact symmetric spaces.

Theorem 4.1. The dot product for double coverings $M \rightarrow M^{\prime \prime}$ and $N \rightarrow N^{\prime \prime}$ has these properties:

(a) $\#_{2} M \leq \#_{2}(M \cdot N)$;

(b) $\#_{2}(M \cdot N) \leq \#_{2}\left(F\left(s_{o}, M\right) \cdot F\left(s_{p}, N\right)\right)+\#_{2}(C M \cdot C N)$, where $C M$ and $C N$ are the centrosomes for the point o of $M$ and its pole and for pof $N$ and its pole;

(c) $\frac{1}{2}\left(\#_{2} M\right)\left(\#_{2} N\right) \leq \#_{2}(M \cdot N) \leq 2\left(\#_{2} M^{\prime \prime}\right)\left(\#_{2} N^{\prime \prime}\right)$.

The 2-numbers has several other links and some nice applications to several ares of mathematics (see e.g. $[13,15,19,22,23,24,25,28,29,30,31,32,33])$. For the most recent survey on 2-numbers, please refer to the survey article [8].

\section{Main theorem}

Let $M$ be a compact symmetric space. Then each polar $M_{+}$of a point $o \in M$ is invariant under the action of the isotropy $K$ at $o$ (cf. Lemma 3.1). A continuous real-valued function $f: M \rightarrow \mathbb{R}$ of a compact symmetric space $M=G / K$ is called isotropic if, for each element $\tau$ in the isotropy subgroup $K$ at $o \in M$ and for each antipodal point $\bar{o}$ of $o$, we have $f(\sigma(\bar{o}))=f(\bar{o})$.

The main result of this paper is the following.

Main Theorem. Let $f: M \rightarrow \mathbb{R}$ be a continuous isotropic function of a compact symmetric space $M$ into the real line $\mathbb{R}$. Then $f$ carries some maximal antipodal set of $M$ to the same point in $\mathbb{R}$, whenever $M$ is one of the following spaces: 
Spheres; the projective spaces $\mathbb{F} P^{n}(\mathbb{F}=\mathbb{R}, \mathbb{C}, \mathbb{H})$; the Cayley plane FII; the exceptional spaces $E I V ; E I V^{*} ; G I$; and the exceptional Lie group $G_{2}$. Consequently, the function $f: M \rightarrow \mathbb{R}$ carries an antipodal set of $M$ with $\#_{2} M$ elements to the same point in $\mathbb{R}$.

Proof. If $\operatorname{dim} M=1$, then $M$ is a circle. Although, in this case the result follows from the Borsuk-Ulam theorem, however for completeness we provide a simple proof for this case.

Without loss of generality, we may assume that $M$ is the unit circle in $\mathbb{R}^{2}$ centered at the origin. For the continuous real-valued function $f: M \rightarrow \mathbb{R}$, we put

$$
g(\mathbf{x})=f(\mathbf{x})-f(-\mathbf{x}), \quad \forall \mathbf{x} \in M \subset \mathbb{R}^{2} .
$$

Then $g$ is a continuous odd function. So we have $g(-\mathbf{x})=-g(\mathbf{x})$. Let $\mathbf{x}_{0}$ be an arbitrary given point in $M$. If we have $g\left(\mathbf{x}_{0}\right)=0$, then $f\left(\mathbf{x}_{0}\right)=f\left(-\mathbf{x}_{0}\right)$ holds. Thus $f$ must carry the pair $\left\{\mathbf{x}_{0},-\mathbf{x}_{0}\right\}$ of antipodal points of $M$ to the same point. On the other hand, it follows from (4.1) that $\left\{\mathbf{x}_{0},-\mathbf{x}_{0}\right\}$ is a maximal antipodal set of $M$. Consequently, we have the desired result.

If $g\left(\mathbf{x}_{0}\right) \neq 0$, without loss of generality we may assume that $g\left(\mathbf{x}_{0}\right)>0$. Since $g$ is odd, we have $g\left(-\mathbf{x}_{0}\right)<0$. Therefore, it follows from the Intermediate Value Theorem that there exists a point $\mathbf{y}$ between $\mathbf{x}_{0}$ and $-\mathbf{x}_{0}$ on which $g(\mathbf{y})=0$. Consequently, the Main Theorem holds for $\operatorname{dim} M=1$.

Next, let us assume that $\operatorname{dim} M \geq 2$ and also $M$ is one of the following spaces: Spheres; the projective spaces $\mathbb{F} P^{n}(\mathbb{F}=\mathbb{R}, \mathbb{C}$, or $\mathbb{H})$; the Cayley plane $F I I$; the exceptional spaces $E I V, E I V^{*}, G I$; and the exceptional Lie group $G_{2}$.

Case (a): $M=S^{n}$. In this case, the Main Theorem follows from the Borsuk-Ulam theorem and (4.1).

Case (b): $M=\mathbb{R} P^{n}$. In this case, it follows from $[6,12]$ that the 2-number of $\mathbb{R} P^{n}$ is given by

$$
\#_{2} \mathbb{R} P^{n}=n+1 .
$$

Thus every maximal antipodal set of $\mathbb{R} P^{n}$ must consist of $n+1$ mutual antipodal points. Moreover, we have

$$
\mathbb{R} P^{n}=S O(n+1) / S O(n) \times\{ \pm 1\} .
$$

It is well-known that the $n$-sphere $S^{n}$ is a Riemannian double covering of $\mathbb{R} P^{n}$ given by

$$
\pi: S^{n}=S O(n+1) / S O(n) \times\{1\} \rightarrow \mathbb{R} P^{n}=S O(n+1) / S O(n) \times\{ \pm 1\},
$$

which is nothing but the antipodal map, that is, $\pi$ maps each antipodal pair of $S^{n}$ to the same point in $\mathbb{R} P^{n}$. Without loss of generality, we may regard $S^{n}$ as the unit hypersphere of $\mathbb{R}^{n+1}$ centered at the origin of $\mathbb{R}^{n+1}$. Via (5.4), we may regard $\mathbb{R} P^{n}$ as the south hemisphere $\breve{S}^{n}$ of $S^{n}$ such that each antipodal pair in $E$ was mapped to the same point in $\mathbb{R} P^{n}$ via $\pi$.

Let $s$ be the south pole of $S^{n}$. We put $o=\pi(s) \in \mathbb{R} P^{n}$. Then the set of all antipodal points of $o \in \mathbb{R} P^{n}$ is exactly given by $\pi(E)$ which is the only polar of $o$ in $\mathbb{R} P^{n}$ (see [10, Table IV, page 416]).

Now, let $f: \mathbb{R} P^{n} \rightarrow \mathbb{R}$ be a continuous isotropic function. Let $\gamma$ be a circle in $\mathbb{R} P^{n}$ containing $o$. Then there exists an antipodal pair $\{p, q\}$ on $\gamma$ which is mapped by the function $f$ to the same point in $\mathbb{R}$ according to Borsuk-Ulam's theorem. Without loss of generality, we may assume that $q$ is the point $o$. Hence we have $f(o)=f(p) \in \mathbb{R}$ and $p \in \pi(E)=\mathbb{R} P^{n-1}$.

Now, let $A_{2}\left(\mathbb{R} P^{n-1}\right)$ be a maximal antipodal set of the polar $\left(\mathbb{R} P^{n}\right)_{+}(p)$ of $o$ in $\mathbb{R} P^{n}$. Since the isotropy subgroup $K$ of $\mathbb{R} P^{n}$ at $o$ acts transitively on the polar $\left(\mathbb{R} P^{n}\right)_{+}(p)$ of $o$ according to Lemma 3.1, we know that, for each point $r \in A_{2}\left(\mathbb{R} P^{n-1}\right)$, there exists an element $\tau \in K$ such that $\tau(p)=r$. Therefore, after applying the isotropic property of the function $f$ we obtain $f(p)=f(r)$. Consequently, the continuous isotropic function $f$ must carry every point of $A_{2}\left(\mathbb{R} P^{n-1}\right)$ to the same point $f(o)=f(p)$. Therefore the Main Theorem is also true for $M=\mathbb{R} P^{n}$.

Case (c): $M=\mathbb{F} P^{n}$ with $\mathbb{F}=\mathbb{C}$ or $\mathbb{H}$. Since $\mathbb{R} P^{n}$ can be isometrically embedded in $\mathbb{C} P^{n}$ and also in $\mathbb{H} P^{n}$ as a totally geodesic submanifold canonically, it follows from Case $(b)$ that the continuous isotropic function $f: \mathbb{F} P^{n} \rightarrow \mathbb{R}$ carries a maximal antipodal set of $\mathbb{R} P^{n} \subset \mathbb{F} P^{n}$ into the same point in $\mathbb{R}$.

On the other hand, it follows from [12] or from [6] that every maximal antipodal set of $\mathbb{R} P^{n}$ is also a maximal antipodal set of $\mathbb{F} P^{n}$ for $\mathbb{F}=\mathbb{C}$ or $\mathbb{H}$. Consequently, the Main Theorem holds for $M=\mathbb{F} P^{n}$ with $\mathbb{F}=\mathbb{C}$ or $\mathbb{H}$.

Case (d): $M=F I I ; E I V ; E I V^{*} ; G I$ or $G_{2}$. Let $o$ be a given fixed point in $M$. Then it follows from [12] or [10, Table IV, page 416] that $o$ has only one polar $M_{+}$in $M$, whenever $M=F I I ; E I V ; E I V^{*} ; G I$ or $G_{2}$. 
Again, let $\gamma$ be a circle in $M$, then the continuous isotropic function $f: M \rightarrow \mathbb{R}$ carries a pair of antipodal points on $\gamma$ to the same point in $\mathbb{R}$. Again, without loss of generality, we may assume that one of the antipodal point from the antipodal pair is $o$ and the other is $p$. Clearly, the antipodal point $p$ must lie in the polar $M_{+}(p)$ of $o$. Now, since $M_{+}(p)$ is only polar of $o$ in $M$, the same argument as given in Case (b) implies that the continuous isotropic function $f$ carries a maximal antipodal set of $M$ to the same point in $\mathbb{R}$. This completes the proof of the Main Theorem.

Now, we provide a simple example to illustrate that the isotropic condition on $f$ in the Main Theorem is essential.

Example 5.1. Consider the real projective plane $\mathbb{R} P^{2}$. Then there is a double covering $\pi: S^{2} \rightarrow \mathbb{R} P^{2}$. Without loss of generality, we may assume that $S^{2}$ is the unit sphere in $\mathbb{E}^{3}$ centered at the origin of $\mathbb{E}^{3}$. For each realvalued function $f: \mathbb{R} P^{2} \rightarrow \mathbb{R}$, the lift $\hat{f}: S^{2} \rightarrow \mathbb{R}$ of $f$ is an even function via the double covering $\pi$, i.e.,

$$
\hat{f}(-\mathbf{x})=\hat{f}(\mathbf{x}), \quad \forall \mathbf{x}=(x, y, z) \in S^{2} .
$$

Conversely, for every given real-valued even function $g: S^{2} \rightarrow \mathbb{R}$ of $S^{2}, g$ induces a real-valued function $\check{g}: \mathbb{R} P^{2} \rightarrow \mathbb{R}$ of $\mathbb{R} P^{2}$.

Now, if we choose $g: S^{2} \rightarrow \mathbb{R}$ to be $g=(x-y)^{2}$, then $g$ induces a real-valued function $\check{g}: \mathbb{R} P^{2} \rightarrow \mathbb{R}$ which does not carry any maximal antipodal set of $\mathbb{R} P^{2}$ to the same point in $\mathbb{R}$.

\section{Some additional results}

All of the compact symmetric spaces $M$ mentioned in the list of the Main Theorem have single polar for a fixed point $o \in M$. In this section, we discuss the case in which the point $o \in M$ has multiple polars.

Suppose $M$ is a compact connected symmetric space such that $o \in M$ has multiple polars $M_{+}^{1}, M_{+}^{2}, \ldots, M_{+}^{i}$. Let $\hat{M}_{+}$denote the polar of $o \in M$ with the maximal 2-number among all polars of $o$.

Theorem 6.1. Let $f: M \rightarrow \mathbb{R}$ be a continuous isotropic function of a compact symmetric space $M$ into the real line $\mathbb{R}$. If $M$ admits more than one polar, then $f$ carries an antipodal set $S_{M}$ of $M$ consisting of $1+\#_{2} \hat{M}_{+}$points of $M$ to the same point in $\mathbb{R}$.

Proof. This can be done in a similar way as Case (d) in the proof of the Main Theorem; just replacing $M_{+}(p)$ by $\hat{M}_{+}(p)$ for an antipodal point $p \in \hat{M}_{+}$of $o \in M$.

At the end of this paper, we provide some (not all) examples of compact connected symmetric spaces which admit multiple polars (see TABLES 2-5).

For instance, from Theorem 6.1 and TABLE 3 we have the following.

Corollary 6.1. If $f: E_{8} \rightarrow \mathbb{R}$ is a continuous isotropic function of the exceptional Lie group $E_{8}$ into the real line $\mathbb{R}$, then $f$ carries an antipodal set of $E_{8}$ with 392 elements to the same point in $\mathbb{R}$.

Similarly, from Theorem 6.1 and TABLE 5 we also have the following.

Corollary 6.2. If $f: F I \rightarrow \mathbb{R}$ is a continuous isotropic function of the compact symmetric space $F I=F_{4} / S p(3) \cdot S U(2)$ into the real line $\mathbb{R}$, then $f$ carries an antipodal set of FI with 24 elements to the same point in $\mathbb{R}$.

\section{References}

[1] Borel, A. and Serre, J.-P., Sur certains sousgroupes des groupes de Lie compacts. Comm. Math. Helv., 27 (1953), 128-139.

[2] Borsuk, K., Drei Satze uber die n-dimensionale euklidische Sphäre. Fund. Math., 20 (1933), 177-190.

[3] Burns, J. M., Homotopy of compact symmetric spaces. Glasgow Math. J., 34 (1992), no. 2, 221-228.

[4] Cartan, É. Sur une classe remarquable d'espaces de Riemann. Bull. Soc. Math. France, 54 (1926), 214-264.

[5] Chen, B.-Y., Geometry of submanifolds. Marcel Dekker, New York, NY, 1973.

[6] Chen, B.-Y., A new approach to compact symmetric spaces and applications. A report on joint work with Professor T. Nagano. Katholieke Universiteit Leuven, Louvain, 1987.

[7] Chen, B.-Y., The 2-ranks of connected compact Lie groups. Taiwanese J. Math., 17 (2013), no. 3, 815-831.

[8] Chen, B.-Y., Two-numbers and their applications - a survey. preprint, 2017.

[9] Chen, B.-Y. and Nagano, T., Totally geodesic submanifolds of symmetric spaces. I. Duke Math. J., 44 (1977), 745-755. 
[10] Chen, B.-Y. and Nagano, T., Totally geodesic submanifolds of symmetric spaces II. Duke Math. J., 45 (1978), no. 2, 405-425.

[11] Chen, B.-Y. and Nagano, T., Un invariant géométrique riemannien. C. R. Acad. Sci. Paris Sér. I Math., 295 (1982), no. 5, 389-391.

[12] Chen, B.-Y. and Nagano, T., A Riemannian geometric invariant and its applications to a problem of Borel and Serre. Trans. Amer. Math. Soc., 308 (1988), no. 1, 273-297.

[13] Console, S., Geodesics and moments maps of symmetric R-spaces. Dipartimento di Matematica - Universit’a di Torino Quaderno N. 25.

[14] Helgason, S.. Differential geometry, Lie groups and symmetric spaces. Academic Press, New York, 1978.

[15] Ikawa, O., Tanaka, M. S. and Tasaki, H., The fixed point set of a holomorphic isometry, the intersection of two real forms in a Hermitian symmetric space of compact type and symmetric triads. Internat. J. Math., 26 (2015), no. 6, 1541005, 32 pp.

[16] Lyusternik, L. A. and Fet, A. I., Variational problems on closed manifolds. Doklady Akad. Nauk SSSR (N.S.), 81 (1951), 17-18.

[17] Lyusternik, L. A. and Shnirel'man, S., Topological Methods in Variational Problems. Trudy Inst. Math. Mech., Moscow State Univ, Moscow, 1930.

[18] Matoušek, J., Using the Borsuk-Ulam theorem. Springer-Verlag, Berlin, 2003.

[19] Nagano, T., The involutions of compact symmetric spaces. Tokyo J. Math., 11 (1988), 57-79.

[20] Nagano, T., The involutions of compact symmetric spaces, II. Tokyo J. Math. 15 (1992), 39-82.

[21] Rotman, J. J., An introduction to algebraic topology. Springer-Verlag, 1988.

[22] Sanchez, C. U., The invariant of Chen-Nagano on flag manifolds. Proc. Amer. Math. Soc., 118 (1993), no. 4, 1237-1242.

[23] Sanchez, C. U., The index number of an R-space: an extension of a result of M. Takeuchi's. Proc. Amer. Math. Soc., 125 (1997), no. 3, 893-900.

[24] Sanchez, C. U. and Giunta, A., The projective rank of a Hermitian symmetric space: a geometric approach and consequences. Math. Ann., 323 (2002), no. 1, 55-79.

[25] Sanchez, C. U., Cali, A. L. and Moreschi, J. L., Spheres in Hermitian symmetric spaces and flag manifolds. Geom. Dedicata , 64 (1997), no. 3, 261-276.

[26] Steinlein, H., Borsuk's antipodal theorem and its generalizations and applications: a survey. Méthodes topologiques en analyse non linéaire. Sém. Math. Supér. Montréal, Sém. Sci. OTAN (NATO Adv. Study Inst.), 95 (1985), 166-235.

[27] Takeuchi, M., Two-number of symmetric $R$-spaces. Nagoya Math. J., 115 (1989), 43-46.

[28] Tanaka, M. S., Antipodal sets of compact symmetric spaces and the intersection of totally geodesic submanifolds. Differential geometry of submanifolds and its related topics, 205-219, World Sci. Publ., 2014.

[29] Tanaka, M. S. and Tasaki, H., The intersection of two real forms in Hermitian symmetric spaces of compact type. J. Math. Soc. Japan, 64 (2012), no. 4, 1297-1332.

[30] Tanaka, M. S. and Tasaki, H., Antipodal sets of symmetric R-spaces. Osaka J. Math., 50 (2013), no. 1, 161-169.

[31] Tasaki, H., The intersection of two real forms in the complex hyperquadric. Tohoku Math. J., 62 (2010), no. 3, 375-382.

[32] Tasaki, H., Antipodal sets in oriented real Grassmann manifolds. Internat. J. Math., 24 (2013), no. 8, 1350061, 28 pp.

[33] Tasaki, H., Estimates of antipodal sets in oriented real Grassmann manifolds. Internat. J. Math., 26 (2015), no. 6, 1541008, 12 pp.

\section{Affiliations}

\section{BANG-YEN CHEN}

AdDrEss: Michigan State University, Department of Mathematics, 619 Red Cedar Road, East Lansing, Michigan 48824-1027, U.S.A.

E-MAIL: bychen@math.msu.edu

ORCID ID : orcid.org/0000-0002-1270-094X 
Table 1. Compact symmetric spaces $M$ with single polar

\begin{tabular}{r|r|r}
\hline Compact symmetric space $M$ & polar $M_{+}$ & $\#_{2} M$ \\
\hline$S^{n}$ & a point & 2 \\
\hline $\mathbb{F} P^{n}(\mathbb{F}=\mathbb{R}, \mathbb{C}, \mathbb{H})$ & $\mathbb{F} P^{n-1}$ & $n+1$ \\
\hline$E I V$ & $F I I$ & 4 \\
\hline$E I V^{*}$ & $F I I$ & 4 \\
\hline$F I I$ & $S^{8}$ & 3 \\
\hline$G I$ & $S^{2} \cdot S^{2}$ & 7 \\
\hline$G_{2}$ & $G I$ & 8 \\
\hline
\end{tabular}

Table 2. Classical group manifolds with multiple polars

\begin{tabular}{r|r|r|r}
\hline Classical group $M$ & $\#_{2} M$ & $\hat{M}_{+}$ & $\#_{2} \hat{M}_{+}$ \\
\hline$S U(n)$ & $2^{n-1}$ & $G_{\left[\frac{n}{2}\right]}\left(\mathbb{C}^{n}\right)$ & $\left(\begin{array}{c}n \\
{\left[\frac{n}{2}\right]}\end{array}\right)$ \\
\hline$S O(n)$ & $2^{n-1}$ & $G_{\left[\frac{n}{2}\right]}\left(\mathbb{R}^{n}\right)$ & $\left(\begin{array}{c}n \\
{\left[\frac{n}{2}\right]}\end{array}\right)$ \\
\hline$S p(n)$ & $2^{n}$ & $G_{\left[\frac{n}{2}\right]}\left(\mathbb{H}^{n}\right)$ & $\left(\begin{array}{c}n \\
{\left[\frac{n}{2}\right]}\end{array}\right)$ \\
\hline
\end{tabular}

Table 3. Exceptional group manifolds with multiple polars

\begin{tabular}{r|r|r|r}
\hline Exceptional group $M$ & $\#_{2} M$ & $\hat{M}_{+}$ & $\#_{2} \hat{M}_{+}$ \\
\hline$E_{6}$ & $2^{6}$ & $E I I$ & 36 \\
\hline$E_{7}$ & $2^{7}$ & $E V I$ & 63 \\
\hline$E_{7}^{*}$ & $2^{8}$ & $E V^{*}$ & 164 \\
\hline$E_{8}$ & $2^{9}$ & $E V I I I$ & 391 \\
\hline$F_{4}$ & $2^{5}$ & $F I$ & 28 \\
\hline
\end{tabular}

Table 4. Classical symmetric spaces with multiple polars

\begin{tabular}{|c|c|c|c|}
\hline Classical symmetric space $M$ & $\#_{2} M$ & $\hat{M}_{+}$ & $\#_{2} \hat{M}_{+}$ \\
\hline$A I(n)=S U(n) / S O(n), n=4 k, 4 k+1,4 k+2$ & $2^{n-1}$ & $G_{2 k}\left(\mathbb{R}^{n}\right)$ & $\left(\begin{array}{c}n \\
2 k\end{array}\right)$ \\
\hline$A I(n)=S U(n) / S O(n), n=4 k+3$ & $2^{n-1}$ & $G_{2 k+2}\left(\mathbb{R}^{n}\right)$ & \\
\hline$A I I(n)=S U(n) / S p(n), n=4 k, 4 k+1,4 k+2$ & $2^{n-1}$ & $G_{2 k}\left(\mathbb{H}^{n}\right)$ & \\
\hline$A I I(n)=S U(n) / S p(n), n=4 k+3$ & $2^{n-1}$ & $G_{2 k+2}\left(\mathbb{H}^{n}\right)$ & \\
\hline$G_{\left[\frac{n}{2}\right]}\left(\mathbb{F}^{n}\right), \mathbb{F}=\mathbb{R}, \mathbb{C}, \mathbb{H}, n \geq 3$ & $\left(\begin{array}{c}n \\
{\left[\frac{n}{2}\right]}\end{array}\right)$ & $G_{\left[\frac{n}{4}\right]}\left(\mathbb{F}^{\left[\frac{n}{2}\right]}\right) \times G_{\left[\frac{n}{2}\right]-\left[\frac{n}{4}\right]}\left(\mathbb{F}^{n-\left[\frac{n}{2}\right]}\right)$ & $\left(\begin{array}{c}{\left[\frac{n}{2}\right]} \\
{\left[\frac{n}{4}\right]}\end{array}\right)\left(\begin{array}{c}n-\left[\frac{n}{2}\right] \\
{\left[\frac{n}{2}\right]-\left[\frac{n}{4}\right]}\end{array}\right)$ \\
\hline$C I(n)=S p(n) / U(n)$ & $2^{n}$ & $G_{r}\left(\mathbb{C}^{n}\right), r=\left[\frac{n}{2}\right]$ & $\left(\begin{array}{l}n \\
r\end{array}\right)$ \\
\hline$D I I I(n)=S O(2 n) / U(n), n=4 k, 4 k+1,4 k+2$ & $2^{n-1}$ & $G_{2 k}\left(\mathbb{C}^{n}\right)$ & $\left(\begin{array}{c}n \\
2 k\end{array}\right)$ \\
\hline $\operatorname{DIII}(n)=S O(2 n) / U(n), n=4 k+3$ & $2^{n-1}$ & $G_{2 k+2}\left(\mathbb{C}^{n}\right)$ & $\left(\begin{array}{c}n \\
n \\
2 k+2\end{array}\right)$ \\
\hline
\end{tabular}


Table 5. Exceptional symmetric spaces with multiple polars

\begin{tabular}{r|r|r|r}
\hline & & & \\
Exceptional symmetric space $M$ & $\#_{2} M$ & $\hat{M}_{+}$ & $\#_{2} \hat{M}_{+}$ \\
\hline$E I$ & 64 & $C I(4)^{*}$ & 36 \\
\hline$E I I$ & 36 & $S^{2} \cdot G_{3}\left(\mathbb{C}^{6}\right)$ & 20 \\
\hline$E I I I$ & 27 & $\widetilde{G}_{2}\left(\mathbb{R}^{10}\right)$ & 10 \\
\hline$E V$ & 128 & $G_{4}\left(\mathbb{C}^{8}\right)^{*}$ & 35 \\
\hline$E V I I$ & 56 & $E I I I$ & 27 \\
\hline$F I$ & 28 & $S^{2} \cdot C I(3)$ & 23 \\
\hline
\end{tabular}

